\title{
Manual de Terapia Nutricional Enteral de Alta Hospitalar Elaboração e Validação de um Instrumento
}

\author{
Edilayne Gomes Boto ${ }^{1}$, Antonia Smara Rodrigues Silva², Letícia Bandeira Mascarenhas ${ }^{3}$, Francisco Valdicélio Ferreira ${ }^{4}$ \\ Mauro Vinicius Dutra Girão ${ }^{5}$, Lia Albuquerque Mendes $^{6}$, Renata Vasconcelos Ferreira? ${ }^{7}$ Lélia Sales de Sousa ${ }^{8}$
}

\begin{abstract}
RESUMO
O suporte nutricional enteral alcançou importante popularidade nos últimos anos, configurando-se como uma alimentação mais fisiológica quando comparada com a nutrição parenteral, sendo o profissional nutricionista o responsável pela prescrição e adequação dietoterápica. Nesta perspectiva, cabe salientar que os cuidados no ambiente domiciliar se configuram como um desafio para este tipo de terapia, observando-se que a elaboração de tecnologias em saúde auxilia na qualificação e aprimoramento do cuidado sob as condições de saúde do paciente e configura-se como importante ferramenta para potencializar a atenção à saúde. Neste sentido, o presente trabalho objetiva elaborar e validar um Manual de orientação para dieta enteral de alta hospitalar para os profissionais da categoria de Nutrição de um Hospital de Ensino. O manual perante os objetivos, conteúdo e relevância para a área hospitalar quanto às imagens, forma de apresentação e conteúdo pertinentes aos cuidados clínicos, apresenta IVC global de 0,98 , sendo classificado como ótimo, tornando-se relevante para atividades de educação e saúde para o nutricionista e usuário de saúde durante a alta hospitalar.
\end{abstract}

Palavras-chave: Manual. Nutrição enteral. Educação em saúde.

\section{MANUAL OF ENTERAL NUTRITION THERAPY FOR HOSPITAL DISCHARGE:} DEVELOPMENT AND VALIDATION OF AN INSTRUMENT

\begin{abstract}
The enteral nutrition support reached important popularity in recent years, configuring it as a more physiological feeding when compared with parenteral nutrition, being the nutritionist professional responsible for dietary prescription and adequacy. In this perspective, it is noteworthy that the care in the home environment are configured as a challenge to this type of therapy, and the elaboration of health technologies that assist in the qualification and improvement of care under the conditions of the patient's health are configured as an important tool to potencializara health care. In this sense, the present study aims to develop and validate a guidance manual of enteral diet for hospital discharge for the professionals in the category of Nutrition of a Teaching Hospital. The manual forward the objectives, content and relevance to the hospital area regarding images, form of presentation and content relevant to clinical care have CVI of 0.98 , being classified as good, making it relevant to health and education activities for the nutritionist and user of health during hospital discharge.

Keywords: Manual. Enteral nutrition. Health education.
\end{abstract}

Recebido em: 19/2/2018

Alterações requeridas em: 4/1/2019

Aceito em: 25/4/2019

\footnotetext{
${ }^{1}$ Nutricionista pelo Centro Universitário Uninta. Coordenadora da Vigilância Alimentar e Nutricional do Municipio de Bela Cruz - CE. edylanne88@hotmail.com

2 Enfermeira pela Universidade Estadual Vale do Acaraú (UVA). Enfermeira na Secretaria Municipal de Saúde de São Benedito - CE. smarynha@hotmail.com

${ }^{3}$ Farmacêutica Centro Universitário Uninta. Preceptora no Centro Universitário Uninta leticiabmascarenhas@hotmail.com

${ }^{4}$ Nutricionista pelo Centro Universitário Uninta. Coordenador da Vigilância Alimentar e Nutricional do município de Sobral - CE. celionutri@gmail.com

${ }^{5}$ Biólogo pela Universidade Estadual Vale do Acaraú (UVA). Professor no Centro Universitário Uninta. viniciusgirao@gmail.com

${ }^{6}$ Nutricionista pelo Centro Universitário Uninta. Nutricionista na Santa Casa de Misericórdia de Sobral - CE. liamendesnutri@gmail.com

7 Nutricionista pelo Centro Universitário Uninta. Nutricionista na Secretaria Municipal de Saúde de Senador Sá - CE. renatavasconcellos16@outlook.com

${ }^{8}$ Nutricionista pela Universidade Estadual do Ceará (Uece). Professora no Centro Universitário Uninta. lelia.sales@uninta.edu.br
} 


\section{INTRODUÇÃO}

A Terapia Nutricional Enteral (TNE) emerge como uma possibilidade terapêutica capaz de atuar na manutenção ou recuperação do estado nutricional. Tão importante quanto a prescrição da TNE adequada às necessidades do paciente, portanto, é a certeza de que o paciente efetivamente receberá o volume prescrito e terá as informações necessárias à sua administração e manuseio no ambiente hospitalar e domiciliar (ASSIS et al., 2010).

O suporte nutricional enteral é utilizado como uma terapia de rotina em pacientes que apresentam alterações no processo de deglutição, em fases agudas e crônicas, quando o paciente não consegue atingir pelo menos $70 \%$ de suas necessidades nutricionais diárias por via oral, sendo necessário, no entanto, que o trato digestório esteja parcial ou totalmente funcionando (PEROTE; VIEIRA; MEDEIROS, 2014).

Diversos fatores podem contribuir para um suporte nutricional inadequado, como a intolerância individual do paciente à dieta, sub ou superestimação das necessidades diárias, manejo inadequado, dentre outros, apontando, assim, a importância de um acompanhamento contínuo do profissional nutricionista desde a internação hospitalar até a assistência ofertada durante a alta hospitalar para aqueles que farão uso desta terapia em ambiente domiciliar, sendo este último um desafio ao profissional de saúde (STEFANELLO; POLL, 2014).

Em decorrência desse processo, no tocante à sistematização da atenção à saúde e suas interfaces na assistência, emerge a importância de os profissionais buscarem estratégias para o fortalecimento da produção científica em tecnologias à saúde e disponibilizarem novas ferramentas de informação e comunicação, possibilitando o diálogo comunicacional, posto que fazem a mediação entre os conhecimentos obtidos nas diversas áreas do conhecimento e da prática (ÁFIO et al., 2014).

Assim, a construção de Manuais e sua utilização representam um recurso pedagógico prático capaz de contribuir para o processo de ensino-aprendizagem, uma vez que sintetiza os conhecimentos obtidos, apresentando-os de forma prática e didática (BRITO; DREYER, 2003).

Conforme Silva e Silveira (2014), embora haja relatos de orientações nutricionais durante a alta hospitalar sobre a utilização de nutrição enteral, o baixo percentual de pacientes que deixam a unidade de saú- de com algum instrumento elaborado pelo profissional de saúde de referência, dificulta a continuidade do cuidado de forma segura e efetiva.

Tal fato remete à importância da criação de instrumentos que proporcionem ao profissional nutricionista, informações relevantes para a elaboração de uma alta hospitalar especializada, com informações pertinentes ao cuidado nutricional no âmbito domiciliar, sendo estes de importante utilidade como instrumento de educação científica e de ensino-aprendizagem, podendo ser utilizado como fonte de pesquisa por profissionais de saúde.

Com base nessas premissas, o presente estudo objetivou descrever o processo de elaboração e validação de um Manual de orientação para dieta enteral de alta hospitalar para os profissionais da categoria de nutrição de um hospital de ensino.

\section{METODOLOGIA}

Trata-se de uma pesquisa metodológica do tipo descritiva com abordagem quantitativa. $O$ estudo desenvolveu-se em duas fases. A primeira consistiu na elaboração do Manual de terapia nutricional enteral de alta hospitalar, a ser utilizado durante a alta hospitalar pelos profissionais da categoria de nutrição. A segunda tratou-se da análise do Manual pelos nutricionistas sobre o conteúdo da tecnologia desenvolvida ante as suas possíveis contribuições para a sistematização do serviço assistencial de nutrição.

Para caracterização dos participantes da pesquisa, aplicou-se um questionário com assuntos relacionados ao conteúdo específico do trabalho, envolvendo pontos acerca do gênero, idade, tempo de formação, tempo de atuação na área clínica, especialização na área hospitalar ou terapia nutricional.

O período de construção, materialização e avaliação da tecnologia ocorreu no primeiro semestre de 2018. No que concerne ao processo de análise da avaliação dos profissionais em relação à tecnologia elaborada, a mesma se deu mediante a aplicação de um questionário adaptado de Mendes (2015), que avaliava o objetivo, conteúdo e relevância da tecnologia.

O referencial metodológico para a construção do Manual teve como embasamento o esboço de Echer (2005), quando foi realizada uma revisão sistemática da literatura pertinente ao tema em questão. Foram incluídas algumas ilustrações na cartilha baseadas nas informações citadas no Manual, com o objetivo de tornar a leitura didática e de fácil compreensão por parte do público-alvo. 
Na etapa de composição do Manual propriamente dito, foi realizado contato com um profissional da área da comunicação, ao qual foi solicitada a realização de um trabalho de edição do material, seguindo algumas das recomendações propostas por Moreira, Nóbrega e Silva (2003) quanto à linguagem, ilustração e layout.

No que concerne à análise dos dados, o material enviado aos examinadores foi verificado e revisado pela pesquisadora. Como procedimento para análise e produção de dados durante a fase de avaliação, foi criado um banco de dados para agregar o conjunto de informações obtidas por meio dos instrumentos aplicados, sendo postos e quantificados pelo programa Excel 2010.

Os critérios foram avaliados de acordo com a valoração: $A=$ de acordo, $A P=$ acordo em parte, $D=$ desacordo e NA = não se aplica, sendo estes designados aos aspectos referentes ao objetivo, conteúdo e relevância da tecnologia desenvolvida.

Posteriormente utilizou-se o Índice de Validade de Conteúdo (IVC), que mede a concordância dos juízes quanto à representatividade dos itens em relação ao conteúdo em estudo. Como aceitável, considerou-se índice mínimo de 0,80 tanto para avaliação de cada item quanto para avaliação geral do instrumento (TIBÚRCIO et al., 2014).

Esta pesquisa foi submetida ao Comitê de Ética em Pesquisa (CEP) do Centro Universitário Inta (Uninta) (parecer favorável de $n^{\circ}$ 2.413.126), tendo Certificado de Apresentação para Apreciação Ética (CAAE) no 71151517.0.0000.8133.

Os nutricionistas colaboraram de forma esclarecida e voluntária e aceitaram participar da pesquisa com a assinatura do Termo de Consentimento Livre e Esclarecido (TCLE). A pesquisa, no que se refere à sua formulação, apresenta consonância aos princípios estabelecidos pela Resolução 466/12 do Conselho Nacional de Saúde.

\section{RESULTADOS E DISCUSSÃO}

Na amostra, o estudo foi composto por 11 nutricionistas, que foram os avaliadores da tecnologia desenvolvida. Destes, $63,3 \%$ eram do sexo feminino e $36,4 \%$ do sexo masculino.

No que concerne ao perfil destes profissionais, 90,9\% têm período de formação e atuação na área clínica de 1 a 5 anos e 9,1\% apresentam tempo de formação e atuação na área clínica superior há 10 anos. No que se refere à titulação acadêmica, $45,5 \%$ são especialistas e $54,5 \%$ dizem estar realizando especialização, tendo como área de concentração em ambos a Nutrição clínica (95\%) e a Terapia Nutricional (5\%).
Um esboço desenvolvido por Sabba et al. (2014) evidenciou que o profissional nutricionista, após sua saída da Graduação e inserção no mercado de trabalho, percebe a necessidade de dar continuidade aos estudos por meio de uma Pós-Graduação, a fim de obter uma melhor qualificação profissional. Observou-se que a pós-Graduação latu sensu foi a primeira escolha, sendo correspondente a 89,2\%, e a Pós-Graduação strictu sensu (Mestrado) referente a 10,8\%.

No tocante ao processo de capacitação sobre a temática "Terapia Nutricional" no ambiente hospitalar, 45,5\% afirmam ter recebido algum treinamento sobre a referida temática. Em contrapartida, 54,5\% afirmam não ter recebido treinamento na área. Conforme Simões et al. (2017), o treinamento com os funcionários no âmbito da terapia nutricional assume importante relevância para o desenvolvimento dos processos de trabalho no serviço hospitalar, devendo essa prática ser intensificada.

No que corresponde aos conteúdos presentes no Manual, inseriu-se temas referentes à terapia nutricional enteral no ambiente hospitalar, propondo um roteiro que propiciasse a construção de um plano de alta pelos nutricionistas para o usuário de saúde conforme a especificidade clínica e social do mesmo. Os conteúdos editorais abordados são representados no Quadro 1.

Quadro 1 - Conteúdo temático da tecnologia "Manual de terapia nutricional enteral de alta hospitalar" 1 - 0 que é Nutrição enteral?

2 - Tipos de dietas enterais.

3 - Higiene pessoal do cuidador durante a manipulação.

4 - Higiene do ambiente para o preparo da dieta e dos alimentos utilizados.

5 - Cuidados com o usuário durante a administração da dieta.

6 - Cuidados com a oferta hídrica.

7 - Cuidados com a administração de medicamentos.

8 - Recomendações durante intercorrências (obstrução e deslocamento da sonda; presença de intercorrências gastrointestinais como diarreia, constipação intestinal vômitos e náuseas).

Todos os avaliadores analisaram a tecnologia de forma detalhada, contribuindo de maneira significativa para o aperfeiçoamento e validação da mesma. Com relação ao processo de julgamento dos itens que compõem o questionário de avaliação da tecnologia, todos obtiveram concordância dentro do nível estabelecido (IVC $>0,85$ ). Os resultados são apresentados nas Tabelas 1, 2 e 3. 
Tabela 1 - Julgamento dos avaliadores $(n=11)$ sobre os itens do questionário referentes ao item dos objetivos da tecnologia (referem propósitos e metas a serem atingidos com a aplicação da tecnologia na prática do Nutricionista)

\begin{tabular}{|c|c|c|c|c|c|}
\hline & $\begin{array}{c}A \\
(n / \%)\end{array}$ & $\begin{array}{c}\text { AP } \\
(n / \%)\end{array}$ & $\begin{array}{c}D \\
(n / \%)\end{array}$ & $\begin{array}{c}N A \\
(n / \%)\end{array}$ & IVC \\
\hline $\begin{array}{l}1.1 \text { São coerentes } \\
\text { com a prática do } \\
\text { Nutricionista. }\end{array}$ & $10 / 90,9 \%$ & $1 / 9,1 \%$ & - & - & 0,98 \\
\hline $\begin{array}{l}1.2 \text { O nível das infor- } \\
\text { mações propostas } \\
\text { está adequado para } \\
\text { serem efetivados na } \\
\text { prática clínica. }\end{array}$ & $10 / 90,9 \%$ & $1 / 9,1 \%$ & - & - & 0,98 \\
\hline
\end{tabular}

Conforme a Tabela 1, é possível inferir que o Manual, quanto ao objetivo ante a sua aplicabilidade na prática diária do nutricionista, bem como as informações contidas nele, é adequado para a efetivação na prática clínica, apresentando um IVC total de 0,98, explanando, assim, nível de concordância ótimo entre os avaliadores.

Tabela 2 - Julgamento dos avaliadores $(n=11)$ sobre os itens do questionário referentes ao item do conteúdo da tecnologia (forma como a tecnologia educativa é apresentada; isso inclui sua organização geral, sua estrutura e estratégia de apresentação)

\begin{tabular}{|c|c|c|c|c|c|}
\hline & $\begin{array}{c}A \\
(n / \%)\end{array}$ & $\begin{array}{c}\text { AP } \\
(n / \%)\end{array}$ & $\begin{array}{c}D \\
(n / \%)\end{array}$ & $\begin{array}{c}\text { NA } \\
(n / \%)\end{array}$ & IVC \\
\hline $\begin{array}{l}2.1 \text { Os conteúdos } \\
\text { atingem com } \\
\text { precisão a } \\
\text { temática } \\
\text { abordada (Terapia } \\
\text { Nutricional } \\
\text { Enteral) }\end{array}$ & $10 / 90,9 \%$ & $1 / 9,1 \%$ & - & - & 0,98 \\
\hline $\begin{array}{c}2.2 \text { As } \\
\text { informações } \\
\text { apresentadas na } \\
\text { tecnologia estão } \\
\text { corretas }\end{array}$ & $09 / 81,8 \%$ & $02 / 18,2 \%$ & - & - & 0,96 \\
\hline $\begin{array}{l}2.3 \text { As imagens } \\
\text { presentes no } \\
\text { manual condizem } \\
\text { com o conteúdo } \\
\text { presente }\end{array}$ & $09 / 81,8 \%$ & $02 / 18,2 \%$ & - & - & 0,96 \\
\hline $\begin{array}{c}2.4 \text { O conteúdo } \\
\text { corresponde ao } \\
\text { objetivo geral } \\
\text { (orientações sobre } \\
\text { terapia nutricional } \\
\text { enteral para a alta } \\
\text { hospitalar) }\end{array}$ & $11 / 100 \%$ & $0 / 0 \%$ & - & - & 1 \\
\hline
\end{tabular}

Pela Tabela 2 conclui-se que o Manual, no que se refere ao conteúdo abordado e às imagens presentes no mesmo, está condizentes para a assistência nutricional, por apresentar um IVC total de 0,97, elucidando, assim, nível de concordância ótimo entre os juízes.

Tabela 3 - Julgamento dos avaliadores $(n=11)$ sobre os itens do questionário referentes ao item de relevância da tecnologia (características que avaliam o grau de significação das orientações de alta hospitalar apresentadas na tecnologia educativa)

\begin{tabular}{|c|c|c|c|c|c|}
\hline & $\begin{array}{c}A \\
(n / \%)\end{array}$ & $\begin{array}{c}\text { AP } \\
(n / \%)\end{array}$ & $\begin{array}{c}D \\
\text { (n/\%) }\end{array}$ & $\begin{array}{c}\text { NA } \\
\text { (n/\%) }\end{array}$ & IVC \\
\hline $\begin{array}{c}3.1 \text { Os itens } \\
\text { expostos no manual } \\
\text { apresentam aspectos } \\
\text { importantes no } \\
\text { que concerne à alta } \\
\text { hospitalar }\end{array}$ & 11/100\% & 0/0\% & - & - & 1 \\
\hline $\begin{array}{l}\text { 3.2 As orientações } \\
\text { presentes } \\
\text { possibilitam a } \\
\text { construção de uma } \\
\text { alta hospitalar efetiva } \\
\text { no que se refere aos } \\
\text { cuidados pertinentes } \\
\text { à terapia enteral para } \\
\text { serem aplicadas no } \\
\text { cuidado domiciliar }\end{array}$ & $10 / 90,9 \%$ & $1 / 9,1 \%$ & - & - & 0,98 \\
\hline
\end{tabular}

Consoante a Tabela 3 é possível concluir que o Manual, no que diz respeito ao grau de relevância para a alta hospitalar e aos cuidados pertinentes à terapia nutricional, é apropriado para a execução no exercício clínico, apresentando um IVC total de 0,99, aclarando o nível de concordância ótimo entre os avaliadores.

Segundo avaliação global, de acordo com as tabelas 1,2 e 3, no parecer final dos avaliadores acerca do questionário de avaliação do Manual, todos os requisitos obtiveram IVC de 0,98 , explanando nível de concordância entre os avaliadores, sendo categorizado como explicativo, importante e adequado, e considerado, assim, uma ótima ferramenta para a prática do nutricionista quanto à alta hospitalar de pacientes em uso de dieta enteral, sendo capaz de promover ações de educação em saúde no momento da alta hospitalar mediante os cuidados domiciliares.

Costa et al. (2013), ao validarem um manual educativo, encontraram, em seu estudo, resultados semelhantes com IVC global de 0,97 , de modo a constituir seu instrumento e conteúdo presentes, válidos e pertinentes para a práxis profissional.

$\mathrm{O}$ processo de validação da tecnologia contou com a participação dos nutricionistas, público ao qual o Manual era destinado, o que possibilitou o aprimoramento do mesmo, sendo avaliados os aspectos relativos ao objetivo, conteúdo e relevância. 
Tal processo evidencia a relevância e cobertura das observações apontadas pelos especialistas. Diferente de outras formas de validação, esta fundamenta-se no respaldo de especialistas em relação ao conteúdo abordado, sendo referendada por um painel de especialistas que tenham qualificação no conteúdo de interesse (CASTRO; LIMA-JÚNIOR, 2014, p. 14).

Destaca-se que a participação de especialistas tanto em conteúdo quanto em conhecimentos metodológicos sobre construção de materiais educativos, faz com que a tecnologia construída tenha suas lacunas preenchidas pelas especificidades do conhecimento de cada profissional, possibilitando o envolvimento em todo o processo de construção do Manual, contribuindo, assim. para um bom percentual da validade de aparência conforme os estudos citados anteriormente (MENDES, 2015).

De acordo com Oliveira, Lopes e Fernandes (2014), os profissionais de saúde motivam-se com a utilização de ferramentas educativas como apoio pedagógico no aprendizado de pacientes, sendo tal fato um consenso entre os estudiosos. É imprescindível considerar a interação de textos e ilustrações no processo de elaboração dos materiais de educação em saúde.

Conforme Varela et al. (2017), a educação em saúde, realizada por meio de tecnologias em saúde, por exemplo os manuais, favorece maior segurança no processo de cuidar, além de ofertar orientações pertinentes ao paciente e seus familiares, potencializando a recuperação e a manutenção da saúde do mesmo.

Logo, o desenvolvimento de estratégias e meIhorias voltadas à prática assistencial e materiais educativos, por intermédio da linguagem e de abordagem clara, assume importante dimensão na contribuição do processo de comunicação com os usuários de saúde, empoderando-os no processo de autocuidado (ALBUQUERQUE et al., 2016).

Nesta perspectiva, o material construído neste estudo apresenta lacunas que vão ao encontro das necessidades do nutricionista no momento da alta hospitalar, apresentando uma mensagem bem-planejada, correta, compreensível e que otimizam as orientações transmitidas no contexto da educação em saúde, oportunizando e fomentando o diálogo entre profissionais e pacientes no tocante ao processo de cuidado em saúde.

\section{CONCLUSÃO}

Ao final do estudo concluímos que os objetivos propostos foram alcançados, ou seja, foi possibilitada a construção e a validação do Manual, material des- tinado a promover ao nutricionista um roteiro para a elaboração da alta hospitalar ao usuário de saúde em uso de terapia nutricional enteral.

Enquanto limitação do estudo, torna-se necessário ainda a validação por profissionais da comunicação a fim de aperfeiçoar a tecnologia desenvolvida. Cabe ressaltar a importância de uma criação posterior de uma versão do Manual já adaptada para o paciente com informações concernentes ao ambiente domiciliar. Destaca-se, como uma dificuldade encontrada durante o estudo, a carência de publicações de validação de material que auxilie na prática educativa do nutricionista, principalmente com ênfase em Nutrição enteral.

Deste modo, o Manual institui-se como uma tecnologia relevante para as atividades de educação e saúde no momento da alta hospitalar, sendo atribuído com valoração em termos técnicos como apto para aplicabilidade clínica. A versão mobile, on-line e impressa do Manual está disponível para os nutricionistas do hospital de ensino no qual foi desenvolvida a pesquisa.

\section{REFERÊNCIAS}

ÁFIO, A. C. E.; BALBINO, A. C.; ALVES, M. D. S.; CARVALHO, L. V.; SANTOS, M. C. L.; OLIVEIRA, N. R. Analysis of the concept of nursing educational technology applied to the patient. Revista Rene, v. 15, n. 1, p. 158-65, Jan./Feb. 2014.

AlBuRquerque, A. F. L. L.; PINHEIRO, A. K. B.; LINHARES, F. M. P.; GUEDES, T. G. Tecnologia para o autocuidado da saúde sexual e reprodutiva de mulheres estomizadas. Rev. Bras. Enferm., v. 69, n. 6, p. 1.164-117, 2016. Disponível em: http://www.scielo.br/scielo.php?pi$\mathrm{d}=$ S003471672016000601164\&script=sci_abstract\&tln$\mathrm{g}=$ pt. Acesso em: 10 out. 2018.

ASSIS, M. C. S.; SILVA, S. M. R.; LEÃES, D. M.; NOVELLO, C. L.; SILVEIRA, C. R. M.; MELLO, E. D.; BEGHETTO, M. G. Nutrição enteral: diferenças entre volume, calorias e proteínas prescritos e administrados em adultos. Revista Brasileira de Terapia Intensiva, São Paulo, v. 22, n. 9, 2010.

BRITO, S.; DREYER, E. Terapia nutricional: conduta do nutricionista. Campinas: Unicamp, 2003.

CASTRO, A. N.; LIMA-JÚNIOR, E. M. Desenvolvimento e validação de cartilha para pacientes vítimas de queimaduras. Rev. Bras. Queimaduras, v. 13, n. 2, p. 103-113, 2014. Disponível em: chrome-extension://oemmndcbldboiebfnladdacbdfmadadm/http://www.scielo.br/pdf/rlae/v22n4/pt_ 0104-1169-rlae-22-04-00611.pdf. Acesso em: 25 jan. 2018.

COSTA, P. B.; CHAGAS, A. C. M. A.; JOVENTINO, E. S.; DODT, R. C. M.; ORIÁ, M. O. B.; XIMENES, L. B. construção e validação de manual educativo para a promoção do aleitamento materno. Revista da Rede de Enfermagem do Nordeste, v. 14, n. 6, p. 1.160-1.167, 2013. Disponível em: http://www. periodicos.ufc.br/rene/article/view/3732. Acesso em: 25 jan. 2018. 
ECHER, I. C. Elaboração de manuais de orientações para o cuidado em saúde. Revista Latino Americana de Enfermagem. v. 13, p. 754-757, set./out. 2005.

MENDES, J. D. Construção e validação de uma tecnologia educativa sobre triagem neonatal no pré-natal. 2015. Trabalho (Conclusão de Curso) - Universidade Vale do Acaraú, 2015.

MOREIRA, M. F.; NÓBREGA, M. M. L.; SILVA, M. I. T. Comunicação escrita: contribuição para a elaboração de material educativo em saúde. Rev. Bras. Enferm., v. 56, n. 2, p. 184-188, 2003.

OLIVEIRA, S. C.; LOPES, M. V. O.; FERNANDES, A. F. C. Development and validation of an educational booklet for healthy eating during pregnancy. Revista Latino-Americano de Enfermagem, v. 22, n. 4, 2014. Disponível em: http://www.scielo.br/scielo.php?script=sci_arttext\&pi$\mathrm{d}=$ S0104-11692014000400611\&lng=en\&tIng=en. Acesso em 25 jan. 2018.

PEROTE, G. M.; VIEIRA, R. Q.; MEDEIROS, J. L. Nutrição enteral e risco de contaminação microbiológica: uma revisão de literatura. Nutrivisa - Revista de Nutrição e Vigilância em Saúde, v. 1, n. 3, 2014.

SABBA, L. J.; OKIDA, Y.; OLIVEIRA, C. R. A.; COUTINHO, R. M. C.; COELHO, H. D. S. Perfil dos egressos do curso de nutrição da Universidade Paulista de um campus em São Paulo. J Health Sci Inst. v. 32, n. 4, p. 424-427, 2014. Disponível em: https://www.unip.br/presencial/comunicacao/ publicacoes/ics/edicoes/2014/04_out-dez/V32_n4_2014_ p424a427.pdf. Acesso em: 25 jan. 2018.

SILVA, A. C.; SILVEIRA, S. A. Perfil epidemiológico e nutricional de usuários de nutrição enteral. Demetra: Alimentação, Nutrição e Saúde, v. 9, p. 783-794, 2014.
SIMÕES, S. A. R.; KUTZ, N. A.; BARBOSA, M. C.; PORTO, E. F.; SALGUEIRO, M. M. H. O. Dieta enteral prescrita versus dieta infundida. Rev. Fund. Care On-line, v. 9, n. 3, p. 688695, 2017. Disponível em: chrome-extension://oemmndcbldboiebfnladdacbdfmadadm/http://www.seer.unirio.br/ index.php/cuidadofundamental/article/download/5506/ pdf. Acesso em: 25 jan. 2018.

STEFANELLO, M. D.; POLL, F.A. Nutritional status and enteral diet prescribed and received by patients of an intensive care unit. ABCS Health Sciences, v. 39, p. 71-76, 2014.

TIBÚRCIO, M. P.; MELOL, G. S. M.; BALDUÍNOL, L. S. C.; COSTAL, I. K. F.; DIASL, T. Y. A. F.; TORRES, G. V. Validação de instrumento para avaliação da habilidade de mensuração da pressão arterial. Rev. Bras. Enferm., v. 67, n. 4, p. 581587, 2014. Disponível em: http://www.scielo.br/pdf/reben/ v67n4/0034-7167-reben-67-04-0581.pdf. Acesso em: 25 jan. 2018.

VARELA, A. I. S.; ROSA, L. M.; RADUNZ, V.; SALUM, N. C.; SOUZA, A. I. J. Cartilha educativa para pacientes em cuidados paliativos e seus familiares: estratégias de construção. Revista de Enfermagem Ufpe On-line, v. 11, p. 2.955-2.962, 2017. Disponível em: chromeextension://oemmndcbldboiebfnladdacbdfmadadm/https://periodicos.ufpe.br/revistas/revistaenfermagem/article/download/11110/19211. Acesso em: 1ㅇo out. 2018. 